ISSN 1516-3660

Produto \& Produção

Revista quadrimestral

(fevereiro, junho e outubro)

de Engenharia de Produção

enfocando suas diversas

áreas: gerência de produção,

gestão da qualidade,

engenharia de qualidade,

qualidade ambiental,

economia industrial, design,

ergonomia, produção civil e transportes.

\section{EDITORES}

Lia Buarque de Macedo Guimarães, Ph.D., CPE

Flávio Sanson Fogliatto, Ph.D.

\section{EDITOR ADJUNTO}

Cristine Porto Brondani

\section{EDITORES PARA ESTE} NÚMERO ESPECIAL

Edson Pinheiro de Lima PPGEPS/PUCPR

Heitor José Pereira

PPAD/PUCPR

Sérgio Eduardo Gouvêa da

Costa

PPGEPS/PUCPR

Programa de Pós-Graduação em

Engenharia de Produção

Escola de Engenharia

UFRGS

Praça Argentina, $\mathrm{n}^{\circ}$ 9, $2^{\circ}$ andar - sala LOPP

Porto Alegre - RS

Fone: (51) 3316-3948 ou

Fax: (51) 3316-4007

E-mail: pep@ufrgs.br

\section{CONSELHO CIENTÍFICO}

Adiel Teixeira de Almeida UFPE - Depto. de Eng. de Produção

Afonso Carlos Corrêa Fleury USP/POLI

Anamaria de Moraes

PUC-RJ - Programa de Mestrado em Design

André Clementino de O. Santos UEPA-CCNT

Antonio Cezar Bornia UFSC - CTC/EPS

Edson Pinheiro de Lima PUCPR - ETO/PPGEPS

Eugenio Kahn Epprecht PUC-RJ - Depto. de Eng. Industrial

Francisco Soares Másculo UFPB - Depto. de Eng. de Produção

Giovani José C. da Silveira University of Calgaray/CA João Ernesto Escosteguy Castro UFSC - EPS

José Luis Duarte Ribeiro UFRGS - PPGEP

Kazuo Hatakeyama CEFET - PR

Marcelo Marcio Soares UFPE - Departamento de Design

Nei Yoshiriro Soma

ITA - IEC

Patrícia Alcantara Cardoso

$U V V-E S$

Paulo José Adissi

UFPE - PPGEP

Paulo Maurício Selig UFSC - CTC/EPS

Regis da Rocha Motta UFRJ - Depto. de Eng. Industrial

Ricardo Naveiro

UFRJ - Programa de Eng.Produção

Sérgio Eduardo Gouvêa da Costa PUC-PR - Depto. Eng. de Produção

Vanderlí Fava de Oliveira

UFJF - Depto. de Eng. de Produção

Waltair Vieira Machado UFAM-PPGEP 


\section{PQP}

\section{REFEREES}

\section{EDIÇÃO ESPECIAL}

\section{A Revista}

Produto \& Produção

agradece aos colegas que,

Luiz Márcio Spinosa

juntamente com o Conselho

Científico, participaram da

avaliação dos artigos

PUCPR

Marco Antonio Masoller

Eleuterio

PUCPR

publicados neste número.

Adriano Proença

UFRJ

Alfredo Iarozinski Neto

PUCPR

Aline França de Abreu

UFSC

Carlos Olavo Quandt

PUCPR

Maria Terezinha Angeloni SBGC

Marly Monteiro de Carvalho USP

Martius Vicente Rodriguez y

Rodriguez

UFF

Moacir de Miranda Oliveira

Junior

PUC-SP

Célia Cristina Zago

$U F P B$

Neri dos Santos

UFSC

Claudio Reis Gonçalo

Oscar Dalfovo

UNISINOS

FURB

Davi Noboru Nakano

USP

Osiris Canciglieri Junior

PUCPR

Denis Alcides Rezende

Osvaldo Luiz Gonçalves

Quelhas

PUCPR

Edson Pinheiro de Lima

PUCPR

Faimara do Rocio Strauhs

UTFPR

Fernando José Barbin Laurindo USP

Gilberto Dias da Cunha

UFF

Paulo Maurício Selig

UFSC

Ricardo Manfredi Naveiro UFRJ

Robert Carlisle Burnett PUCPR

PUCRS

Heitor José Pereira

PUCPR

Hélio Gomes de Carvalho

UTFPR

Lucia Izabel Czerwonka

Sermann

PUCPR

Luiz Carlos Duclós

PUCPR

Rui Francisco Martins Marçal UTFPR

Sérgio Eduardo Gouvêa da Costa PUCPR

Sérgio Luis da Silva

UFSCar 


\section{EDITORIAL}

\section{Edição especial em gestão do conhecimento}

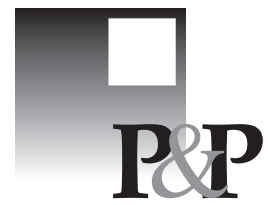

Tem-se observado uma profunda mudança nas relações socioeconômicas dos modernos sistemas produtivos, cenário este que se verifica tanto na sua estrutura, como também na definição do seu papel como agente econômico. Uma série de questões relacionadas com a sustentabilidade, a intangibilidade, a complexidade, a dinâmica, a responsabilidade social e ambiental, estão sendo tratadas com alta prioridade na agenda das empresas, das instituições públicas governamentais e não governamentais. Um modelo que representa este novo conjunto de relações vem sendo desenvolvido, tendo como fundamento o novo paradigma da "Economia do Conhecimento". No âmbito das organizações, esta mudança tem-se refletido na busca constante por novos processos e práticas de Gestão do Conhecimento, esta percebida, cada vez mais, como recurso estratégico. O impacto da Gestão do Conhecimento abrange todos os processos organizacionais e de gestão, relacionando-se tanto a atividades-fim como a atividades-meio. A revista Produto \& Produção, em edição especial, apresenta uma discussão acerca das relações deste novo modelo com as mudanças que estão sendo experimentadas nos campos de atuação da Engenharia de Produção e da Gestão de Operações.

Atendendo ao convite dos co-editores desta edição especial, José Terra desenvolveu um conjunto de reflexões sobre a evolução da gestão do conhecimento no Brasil. Tais reflexões estabelecem um contexto para se discutirem os principais desafios da gestão do conhecimento no âmbito da Engenharia de Produção e da Gestão de Operações. Tendo sido estabelecido um contexto para delimitar a discussão acerca da Gestão do Conhecimento, Davi Nakano e Afonso Fleury constroem um quadro teórico-conceitual na forma de modelos para o estudo do conhecimento organizacional. A partir do contexto estabelecido e do quadro teórico montado, decorre uma série de estudos no nível teórico-conceitual e no nível de aplicação. Os estudos que se desenvolvem a respeito das diretrizes estratégicas para implantação da gestão do conhecimento de Edson Pinheiro, Heitor Pereira, Sérgio Gouvêa, Alex Ferraresi e Marco Busetti; das barreiras cognitivas e suas relações com a ação organizacional de Cláudio Gonçalo; da organização baseada em conhecimento de Simone Paiva, Paulo de Aragão e Sandra Pereira; da construção da qualificação de Mariângela Martinez e Alessandra Rachid; e da formação de comunidades estratégicas de Augusto Cruz Jr., Marly Carvalho, Fernando Laurindo e Marcelo Pessoa, são construções de natureza teórico-conceitual refinadas e/ou validadas através de aplicações em projetos específicos.

Os artigos que complementam esta edição especial tratam de aplicações específicas da Gestão do Conhecimento. Uma das áreas da Engenharia de Produção que atualmente mais concentra trabalhos no tema Gestão do Conhecimento é a Engenharia do Produto. Os trabalhos de Osíris Canciglieri Jr., Robert Young e Fábio Favaretto, de Sérgio da Silva, e de Ricardo Naveiro e Marcos Borges, relacionam-se com essa área, tratando de aspectos relativos ao projeto para a manufatura, ao desenvolvimento de uma infra-estrutura para o processo virtual de desenvolvimento de produto e ao próprio processo criativo de desenvolvimento do produto, respectivamente. Também os sistemas de informação e o seu desenvolvimento, dentro do enfoque da gestão do conhecimento, encontram-se representados no artigo de Denis Rezende e José Guagliardi. $\mathrm{O}$ estudo das redes e arranjos produtivos também vem sendo feito à luz da gestão do conhecimento, como pode ser observado no artigo de Marcos Cavalcanti, Rosa Lima e André Neto. Um setor que vem-se destacando no seu processo de modernização, por adotar uma abordagem voltada à gestão do conhecimento, é o setor de energia. O conceito de gestão estratégica do conhecimento é desenvolvido no trabalho de Edson Pinheiro et al com aplicação em empresa do setor de energia, bem como o trabalho de Dagoberto Almeida e Liliane Fagundes traz uma aplicação da gestão do conhecimento no mapeamento de falhas em concessionárias de energia elétrica. A visão dos serviços, da qualidade e da gestão de projetos e a sua relação com a gestão do conhecimento é explorada no artigo de Denise Bassani, Sonia Nikitiuk, Osvaldo Quelhas e Sérgio França.

Os artigos selecionados para esta Edição Especial em Gestão do Conhecimento da revista Produto e Produção (números 2 e 3 do volume 8 de 2005) formam um todo coerente que visa ao desenvolvimento de aplicações por intermédio do refinamento e validação de frameworks e de modelos conceituais, bem como à geração de novas teorias e modelos, através da experimentação e síntese proporcionadas pelas diferentes aplicações da gestão do conhecimento. Esperamos que esta edição especial estimule o avanço das pesquisas e publicações nesta área temática de fronteira do conhecimento em gestão empresarial, particularmente no que se refere à gestão de operações.

Co-editores edição especial em Gestão do Conhecimento

Edson Pinheiro de Lima (PPGEPS/PUCPR)

Heitor José Pereira (PPAD/PUCPR)

Sérgio Eduardo Gouvêa da Costa (PPGEPS/PUCPR) 

Produto \& Produção, vol. 8, n. 2, p. 05-09, jun. 2005

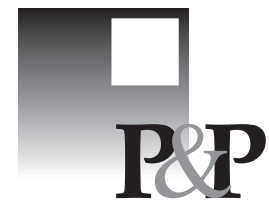

\title{
Reflexões sobre a evolução da Gestão do Conhecimento no Brasil
}

\author{
José Cláudio Cyrineu Terra, Dr. \\ Presidente da TerraForum Consultores \\ www.terraforum.com.br
}

\begin{abstract}
O artigo tem um caráter bastante reflexivo sobre a Gestão do Conhecimento no Brasil. Discute em que medida e de que formas a Gestão do Conhecimento tem influenciado a prática administrativa nas organizações brasileiras. A discussão se fundamenta na experiência acadêmica e prática do autor. As considerações sobre o impacto da Gestão do Conhecimento no Brasil são analisadas principalmente a partir de algumas considerações teóricas da evolução da ciência administrativa e sobre alguns aspectos específicos como
\end{abstract}

Palavras-chave: 7 dimensões; modelos mentais; ciclo do conhecimento.

This is a reflexive paper on the knowledge management in Brazil. It discusses in what extent and how knowledge management has influenced the administration practices into the Brazilian organizations. The debate is based on the academic and practical experience of the author. The considerations on the impact of the knowledge management in Brazil have been analyzed mainly from the theoretical issue of the evolution of the management sciences and of some specific aspects as the communication and information technologies role and their relationship with the productivity index.

Keywords: 7 dimensions; mental models; knowledge cycle.

\section{Introdução}

É evidente que a Internet mudou muita coisa no seio das organizações: fluxos de informação foram dinamizados, distâncias foram encurtadas e a colaboração remota, assíncrona e síncrona, também atingiu patamares muito mais elevados. A Internet é, sem dúvida alguma, uma revolução. A Gestão do Conhecimento tem se beneficiado muito desta revolução, mas vai muito além, pois seu foco é o conhecimento e não simplesmente a informação.

O que significa desenvolver vantagens competitivas duradouras em um mundo ambiente competitivo cada vez mais dependente de inovações e aplicação de conhecimento e informação de valor? Em que medida o conceito de vantagem competitiva tem a ver com produtividade do trabalhador do conhecimento? E em que medida a produtividade do trabalhador do conhecimento tem a ver com as abordagens da Gestão do Conhecimento? Finalmente, o impacto da "onda" da Gestão do Conhecimento pode ser mensurado apenas diretamente pelos programas e métodos específicos que a mesma trouxe consigo?
Argumentamos neste artigo que estas relações são crescentemente verdadeiras e mesmo centrais no contexto da Era da Informação e do Conhecimento. Além disso, verificamos que, intuitivamente, uma série de organizações vem seguindo a lógica Gestão do Conhecimento-Produtividade-Competitividade, mesmo sem explicitar diretamente os conceitos e termos aqui apresentados.

\section{A "onda" da Gestão do Conhecimento}

Há pouco mais de dez anos eram basicamente as empresas globais de consultoria de gestão que contavam com pessoal dedicado e processos estruturados para tratar da Gestão do Conhecimento. Esta realidade mudou. Neste período, um número substancial de empresas, em geral as mais inovadoras e líderes de seus segmentos, passou a reconhecer o conhecimento como fator crítico para a competitividade e a tomar ações consistentes com esta visão. Nos países mais avançados há muitas empresas com processos bem consolidados nesta direção. No Brasil, casos bem consolidados são relativamente recentes. Os casos mais conhecidos e relevantes tiveram início nos últimos dois ou três anos. 
A Gestão do Conhecimento é particularmente avançada e valorizada no setor de professional services (consultoria, serviços de TI, agências de publicidade, grandes escritórios de advocacia, etc.) e de empresas de alta tecnologia (química fina, informática e engenharia), pois estas são baseadas essencialmente em conhecimento. Processos, iniciativas e práticas de Gestão do Conhecimento, longe de serem exclusividade dos segmentos acima mencionados, estão se tornando cada vez mais evidentes também nos setores manufatureiros, indústria de processos contínuos, exploração de recursos naturais e mesmo governo e organizações sem fins lucrativos.

De fato, dez anos atrás quando começamos a pesquisar, pensar e discutir Gestão do Conhecimento havia muito poucos interlocutores na academia e quase ninguém no mundo empresarial. Este cenário mudou e muito. Alguns dizem que Gestão do Conhecimento virou a "onda" no mundo da gestão nos últimos anos. Artigos, palestras e teses se proliferam por todos os cantos do Brasil. Há também um número significativo de empresas com iniciativas com o rótulo de Gestão do Conhecimento. Entre consultores, gerentes e professores há milhares de pessoas apenas no Brasil discutindo, aplicando, gerenciando e divulgando a Gestão do Conhecimento.

Uma série de organizações líderes no Brasil, como Aracruz, Caixa Econômica Federal, Camargo Correa, Banco do Brasil, Editora Abril, Eletrobrás, Embrapa, Natura, Petrobrás, Promon, SABESP, SERPRO e Grupo Votorantim vêem adotando a Gestão do Conhecimento como uma iniciativa deliberada e sistemática. Várias destas organizações contam inclusive com alguém no cargo de Gerente de Gestão do Conhecimento. No exterior - e entre as empresas de consultoria em particular a Gestão do Conhecimento já tem um histórico bem mais avançado. Algumas organizações chegam inclusive a ter cargos como Diretor Mundial de Gestão do Conhecimento ou Chief Knowledge Officer (CKO).

\section{Gestão e Conhecimento}

Tudo isto representa um claro sucesso. Certo? Ou não? Em que medida a Gestão do Conhecimento pode estar associada a bons desempenhos empresariais ou à eficiência da máquina pública? Quais as métricas? Será que a Gestão do Conhecimento pode ser medida? Quais são os casos de sucesso comprovado? Não temos a pretensão de ter a resposta a estas questões. Vamos, no entanto, tentar responder a estas questões a partir de algumas considerações mais amplas sobre gestão e conhecimento. Depois disso, voltamos a falar de Gestão do Conhecimento.

Gestão ou administração é uma das disciplinas mais óbvias e ao mesmo tempo mais complexas. É uma disciplina relativamente fácil de ser ensinada em comparação a outros cursos mais "pesados" como medicina ou engenharia. Esta é uma frase logicamente provocadora. O fato é que gestão envolve muito mais que aprender algumas técnicas e métodos. Gestão envolve uma grande dose de habilidades sociais, relacionamento pessoal, intuição e experiência vivida. Mas não é só isso, gestão envolve também um conjunto de pressupostos, preconceitos e modelos mentais que moldam comportamentos, decisões e ações.

Definir e discutir conhecimento, logicamente, não é também uma tarefa das mais fáceis. Comecemos, por exemplo, tentando definir, enquadrar ou classificar nosso próprio conhecimento. O que sabemos? Quanto do nosso conhecimento é consciente? Quanto do mesmo vive nas cavernas inexploradas do nosso subconsciente? Como nosso conhecimento subconsciente influencia nossas ações? Por que decidimos o que decidimos? Por que fazemos o que fazemos? Qual o nosso grau de liberdade ou livre arbítrio na condução do nosso aprendizado e formação do nosso conhecimento? Quanto da realidade percebemos? Perguntas, perguntas e mais perguntas. Todas altamente complexas e centrais para a definição da nossa condição humana. Por isso mesmo, estas questões têm sido discutidas pelas mentes mais brilhantes da história humana há milhares de anos.

É, pois, no contexto destes dois campos "gestão" e "conhecimento", que surge a "Gestão do Conhecimento". De Nonaka, com seu clássico ciclo SECI, a proponentes de mapeamentos os mais diversos, comunidades, portais e storytelling, há uma miríade de abordagens, práticas e tecnologias que atualmente se encaixam, se refugiam ou se apóiam na Gestão do Conhecimento. O Brasil e suas organizações de ponta têm adotado várias das mesmas práticas, iniciativas e tecnologias das empresas líderes mundiais. É verdade que, invariavelmente, com mais lentidão, entusiasmo e recursos. De qualquer maneira, há uma evolução clara no sentido da implantação de "Boas Práticas de Gestão do Conhecimento". Algumas pesquisas têm sido feitas neste sentido. Nós mesmos participamos, recentemente, de uma ampla pesquisa sobre práticas de Gestão do Conhecimento no setor público (FERREIRA, QUANDT, PACHECO e TERRA, 2005).

\section{Definindo a Gestão do Conhecimento}

Mas, afinal, o que é Gestão do Conhecimento? É uma teoria, uma disciplina, um método, uma abordagem ou a aplicação de um conjunto de tecnologias de informação e comunicação? Nós a definimos como um foco gerencial deliberado, sistemático e organizado nos ativos intan- 
gíveis da organização (aspecto não abordado neste artigo) e, principalmente, na produtividade geral do trabalhador do conhecimento e no desenvolvimento de métodos e ferramentas focadas no conhecimento estratégico e no Ciclo do Conhecimento Organizacional (Figura 1), que envolve atividades relacionadas à Inovação, Codificação, Organização, Compartilhamento, Disseminação e Proteção do Conhecimento.

A eficiência e eficácia deste ciclo, por sua vez, depende de um contexto organizacional amplo. É neste sentido que nosso modelo das 7 Dimensões se insere (TERRA, 2005). O Ciclo do Conhecimento Organizacional depende de ações nas dimensões Estratégia, Processos \& Organização, Cultura Organiza-cional, Políticas de Recursos Humanos, Infra-Estrutura de TI, Métricas e Aprendizado com o Ambiente.

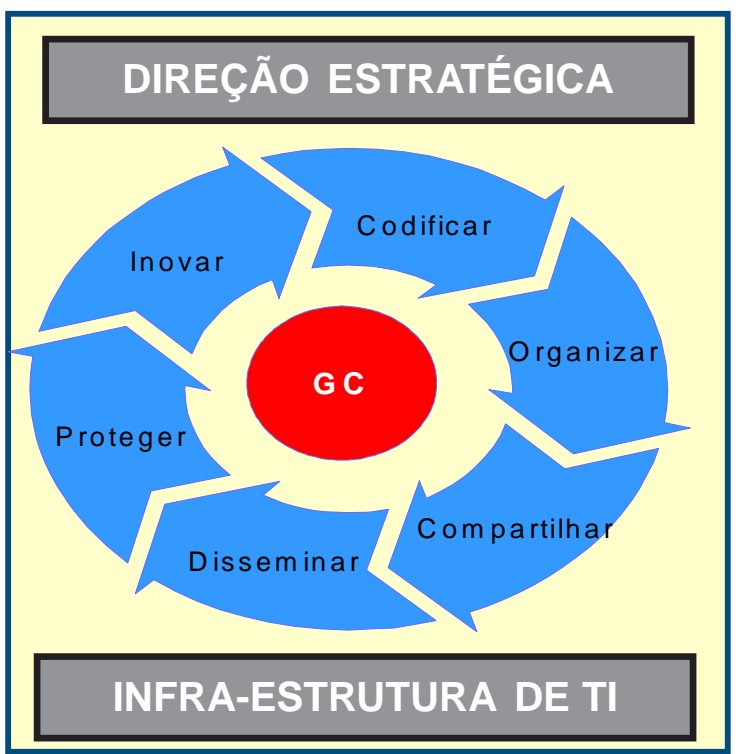

Figura 1 - Ciclo do Conhecimento

Muitas empresas já realizam uma série de iniciativas que se encaixam na definição acima. Em alguns casos, elas se manifestam por iniciativas horizontais, como no caso do desenvolvimento de Intranets, que facilitam o acesso geral à informação, aplicativos e sistemas de comunicação, resultando, quando bem implementada, em um aumento geral de produtividade. Há também iniciativas verticais, normalmente mais estratégicas e focadas em algum processo estratégico, área temática ou grupo de pessoas. Estas últimas se manifestam normalmente por iniciativas como comunidades de prática, banco de melhores práticas ou criação de grupos de inovação. Alguns de seus resultados, mas não todos, são mais facilmente mensuráveis a partir de estudos de caso, que mostram a adoção de novos métodos produtivos, concretização de uma venda difícil ou o desenvolvimento de uma inovação importante.

Em função desta definição ampla, a Gestão do Conhecimento traduz-se na prática a partir de vários tipos de métodos e ferramentas utilizados. Isto é particularmente verdade entre empresas de diferentes portes, distribuição geográfica, setores econômicos e até mesmo estratégia competitiva. Além disso, programas e iniciativas de Gestão do Conhecimento têm sido desenvolvidos com diferentes ênfases nos vários elementos do Ciclo do Conhecimento Organizacional (conforme Figura 1 acima) e na natureza do conhecimento em foco (Tácito ou Explícito $^{1}$ ) que se quer gerenciar. Idealmente, ademais, a Gestão do Conhecimento deveria estar plenamente ancorada na estratégia empresarial. Quando isto ocorre e os esforços descritos acima passam a ser melhor articulados, dizemos que uma organização tem uma iniciativa estruturada de Gestão do Conhecimento ao invés de fragmentos de ações gerenciais que contribuem para a Gestão do Conhecimento.

\section{Gestão do Conhecimento e produtividade}

Ao contrário do trabalhador cujo principal output de seu trabalho depende de seu esforço físico, o trabalhador do conhecimento se alimenta de informação, conhecimento, experiências, contatos e modelos mentais para produzir essencialmente resultados intangíveis como decisões, relatórios, designs e inovações. O processo em si, neste caso, é invisível, se passa na cabeça dos indivíduos. Isto é verdade, tanto para um engenheiro que projeta uma máquina sofisticada, como para um operário qualificado que precisa monitorar e regular múltiplos sistemas e cujo trabalho diário se resume à tomada de decisões. Cada vez mais, as atividades geradoras de valor econômico dependem, em maior ou menor grau, de inputs de conhecimento.

A produtividade dos trabalhadores do conhecimento é, portanto, em boa medida, o que torna algumas empresas competitivas, inovadoras e líderes. Isto é uma verdade absoluta no caso de empresas cujo produto é intangível por natureza, como no caso de consultorias,

1 Conhecimento tácito sendo compreendido por aquele conhecimento de difícil externalização e cuja frase célebre que o sintetiza é "sabemos muito mais do que podemos dizer". É o conhecimento que se adquire normalmente ao longo do tempo a partir de múltiplas experiências vividas. Já o conhecimento explícito é aquele mais associado ao conhecimento embutido em produtos, processos, fórmulas, software, documentos, etc. É um conhecimento muito mais facilmente transmitido e copiado do que o tácito. Os conceitos de conhecimento tácito e explícito são centrais para a Teoria do Conhecimento e foram muito bem explorados nos trabalhos clássicos de Michael Polany (1958) e Nonaka e Takeuchi (1995). Mais recentemente, este próprio autor teve seu trabalho sobre o mesmo tema premiado em congresso na França (TERRA E ANGELONI, 2003). 
agências de publicidade, empresas de treinamento, etc. No caso de empresas onde a receita advém da venda de produtos tangíveis como minério, aço, móveis, computadores, telefones, etc., o papel dos processos intangíveis é cada vez mais relevante. O trabalho físico e os processos físicos, que muitas vezes materializam o resultado do trabalho mental, são geralmente "commodities" que não garantem diferenciação e retornos elevados. É o conhecimento embutido "nas coisas" que gera valor econômico.

É neste contexto de foco nas atividades geradoras de valor e diferenciadoras que enxergamos o papel da Gestão do Conhecimento nas organizações. Ela deve, no final das contas, prover meios que alavancam o output dos trabalhadores do conhecimento. Como isto pode acontecer? Em termos gerais, desenhando um ambiente favorável à criatividade, inovação, iniciativa, aprendizado constante e compartilhamento de informação e conhecimento. Envolve também o provimento de mecanismos de publicação e acesso à informação seletiva, validada, personalizada e estratégica. Finalmente, a Gestão do Conhecimento envolve mecanismos para a rápida identificação de talentos, expertises e estímulo para que pares se encontrem, troquem idéias, experiências e inovem.

Isto quer dizer, ademais, que os indivíduos afetados ou diretamente envolvidos pela Gestão do Conhecimento deverão adquirir e aprimorar constantemente suas habilidades relacionadas à busca, análise, validação, publicação, colaboração e disseminação de informação e conhecimento. Ser produtivo na Era da Informação e do Conhecimento requer menos foco no exercício da memória, e mais no exercício das habilidades de processamento, colaboração, inovação e decisão em torno da informação e do conhecimento.

Embora saibamos intuitivamente distinguir uma pessoa produtiva de uma pessoa não produtiva, não temos métricas padrão similares àquelas utilizadas para o trabalho repetitivo e rotineiro $(\mathrm{kg} /$ hora, toneladas/ano, etc.). Logicamente, não faz sentido medir coisas como relatórios/hora ou decisões/dia. Além disso, um outro aspecto relacionado à mensuração da produtividade do trabalhador do conhecimento é que, diferentemente do trabalho de natureza estritamente física, os resultados são obtidos de maneira coletiva, complexa e integrada à ação de vários outros indivíduos.

Isto tudo significa que boa parte das ações gerenciais no sentido de melhorar a produtividade do trabalho intelectual são, em grande medida, uma constante aposta no sentido de que a melhoria das condições de trabalho e o acesso à informação e ao conhecimento produzirão melhores decisões, uso mais efetivo de boas práticas e, de tempos em tempos, geração de inovações significativas. As métricas tendem a ser, portanto, muito mais qualitativas do que quantitativas. No final das contas, o que vale é o valor gerado ou percebido pelas ações, decisões e inovações dos trabalhadores do conhecimento.

Esta é uma situação que gera um grande desconforto para um grande número de pessoas que cresceram em um mundo industrial no qual os processos podem ser decompostos em sub-processos bem específicos e o controle dos inputs, processos e outputs de cada parte são muito mais óbvios e diretos. O desconforto advém do fato que investir na produtividade do trabalhador do conhecimento, ou em conhecimento em um sentido mais amplo, é sempre um risco e com resultados tanto positivos, como negativos, por vezes, bastante imprevisíveis. Um exemplo simples, mas que antecede a discussão em torno da Gestão do Conhecimento ilustra esta questão: imagine uma empresa que financia um MBA para 30 de seus executivos. Que garantia esta empresa tem que tal investimento vai gerar retorno? Nenhum, principalmente de forma claramente mensurável. Mais ainda, o que significa retorno neste caso? Apesar destas questões, é sabido, no entanto, que empresas líderes e admiradas investem, invariavelmente, bem acima da média na qualificação de seus profissionais.

\section{Os resultados da Gestão do Conhecimento no Brasil}

Entender a situação da Gestão do Conhecimento no Brasil se beneficia, evidentemente, de trabalhos baseados em pesquisa de campo e levantamentos estatísticos. Se voltarmos às discussões acima sobre "Gestão" e "Conhecimento" veremos, no entanto, que tão importante quanto as métricas específicas de qualquer tipo de iniciativa de Gestão do Conhecimento (que também são importantes), são os resultados obtidos na mudança de modelos mentais de nossos gestores. Estes, apesar de críticos, não podem ser medidos por meio de métricas econômicas ou de produtividade.

Enfim, nossa percepção é que a "disciplina” Gestão do Conhecimento em suas múltiplas facetas está contribuindo de maneira efetiva para a produtividade do trabalhador brasileiro e para a evolução dos modelos gerenciais das organizações brasileiras. Em alguns casos, isto significa uma mudança significativa do foco e atenção aos processos de aprendizado organizacional a partir de uma base mais sólida sobre teorias de gestão da informação e também de teorias sobre aprendizado, criatividade e conhecimento. Tem envolvido também mudanças nos modelos mentais quanto à percepção sobre as verdadeiras fontes e formas de geração e distribuição de riqueza e utilização de recursos escassos. Logicamente, métodos e tecnologias para melhor gestão de informação com foco no aprendizado também foram introduzidos nos últimos anos. 
Para os céticos o copo está totalmente vazio: "mostremme os resultados concretos da Gestão do Conhecimento: Quanto de riqueza, eficiência e produtividade foram resultados da Gestão do Conhecimento nos últimos anos?" Já os entusiastas dirão que o copo está totalmente cheio. As empresas líderes mudaram significativamente seus modelos de gestão, mensuração de resultados e adotaram várias práticas e tecnologias modernas nos últimos anos. A realidade, logicamente, está no meio do caminho. A teoria e prática administrativa não evoluem, com raras exceções (método científico no início do século XX), por meio de revoluções, mas sim por meio de evoluções cumulativas que se incorporam ao dia-a-dia dos gestores, que nem sabem o que sabem e porque sabem o que sabem.

Conhecimento, diferentemente de informação, é construído socialmente, depende de múltiplas experiências captadas por nossos sentidos e se revela principalmente na ação e na decisão. Por outro lado, pode levar muito tempo para se revelar e de um momento para outro "Eureka". Não é, portanto, mera coincidência que empresas que investem mais fortemente em treinamento e em inovação de produtos e serviços também tendam a ser mais receptivas para a Gestão do Conhecimento. Elas sabem ou intuem que o investimento no aprendizado individual e coletivo eventualmente gera mais produtividade e competitividade. Há um certo consenso que o uso da criatividade e a rápida apropriação do conhecimento humano é o grande diferencial entre nações desenvolvidas e em desenvolvimento, entre empresas líderes e inovadoras e aquelas seguidoras e conservadoras. Por outro lado, há um velho ditado em gestão que diz: "Só se gerencia o que se mede". A este ditado contrapomos uma citação não menos célebre de Einstein: "Nem tudo que conta pode ser medido; e nem tudo que pode ser medido, conta". Gerir conhecimento não é simples e nem facilmente mensurável. É, porém, a grande questão gerencial que enfrentamos. Sabemos que quando bem realizada, ela afeta a produtividade dos trabalhadores do conhecimento, principalmente ao reduzir o tempo que estes despendem em atividades de baixo valor agregado e ao prover condições para que os mesmos tenham acesso ao melhor em termos de informação e conhecimentos disponíveis, para decidir, agir ou inovar.

A Gestão do Conhecimento é muito mais uma consolidação de conceitos e um pensar muito mais reflexivo sobre as características e o papel do conhecimento individual e organizacional para o desempenho das organizações. Os milhares de estudiosos e praticantes da Gestão do Conhecimento estão, neste sentido, contribuindo para a evolução da prática administrativa das empresas brasileiras, mesmo que de forma indireta ao influenciar o modelo mental dominante em nossas organizações.

\section{Referências}

FERREIRA, F.; QUANDT, C.; PACHECO, F. e TERRA,

J. Gestão do Conhecimento na Administração

Pública. IPEA, Texto para Discussão N 1095, 2005.

NONAKA, I. e TAKEUCHI, H. The Knowledge-creating company: how Japanese companies create the dynamics of innovation. Oxford University Press, New York, 1995.

POLANY, M. Personal Knowledge: Towards a PostCritical Philosophy. Harper Torchbooks, 1958.

TERRA, J. C. Gestão do Conhecimento: o grande desafio empresarial. 5. ed. Negócio Editora, 2005.

TERRA, J. C. e ANGELONI, T. "Understanding the difference between Information Management and Knowledge Management", presented at The 12th International Conference on Management of Technology, Nancy, France, May 12-13, 2003. 
